\title{
Postoperative Jejuno-Jenunal Intussusception - A Rare Complication Following Total Gastrectomy
}

\author{
Satya Prasad Samantaray, MCh. , Dilip Chakravarty K, MS ${ }^{2}$, Mounika Kilari, MD², \\ Praveen Kumar AC, DM ${ }^{4}$ \\ ${ }^{1}$ Department of Surgical Gastroenterology, SCB Medical College, Cuttack, Odisha, India \\ ${ }^{2,3,4}$ Department of Gastroenterology, Vydehi Institute of Medical Sciences and Research Centre, Bangalore, India
}

Note: Author 1 and 2 Equally Contributed in this Case Report

\begin{abstract}
Intussusception commonly occurs in pediatric age group where the etiology is idiopathic, in contrast to adults, where aetiology if often found. Ileocolic intuscusception is the most common type, but colocolic type is common in adults. Intussusception is a common cause of intestinal obstruction, but its occurrence in the post-operative period after bowel surgery is infrequent except for retrograde jejuno-gastric intussusception. We present here an unusual case of jejuno-jejunal intussusception in a 60 year old man who had undergone total gastrectomy with D2 nodal dissection and Roux-en-Y oesophagojejunostomy presenting with features of subacute intestinal obstruction, gastrointestinal bleeding and sepsis 2 weeks after surgery. He was evaluated by barium meal follow through examination. Surgery was offered in the fourth post-operative week in the form of manual reduction of the intussusception and peritoneal toileting after 2 days of conservative trial. Follow-up is necessary as possibility of recurrence cannot be excluded.
\end{abstract}

Keywords: Intussusception, Oesophagojejunostomy, Gastrectomy

\section{Introduction}

Intussusception involves telescoping of a segment of bowel into an adjacent segment, leading to obstruction, inflammation, and possible ischemia (1). Post-operative jejuno-jejunal intussusception is one of the rarest complications of the previous gastric surgery. The incidence is reported to be less than $0.1 \%$ (2). This complication can occur at any time after Billroth II gastrectomy or gastrojejunostomy (3). The clinical presentation of intussusception in adults can be nonspecific, unlike children, leading to a delay in the diagnosis. In contrast to pediatric intussusceptions, which are managed non-operatively with air contrast enemas, treatment in adults is exploratory laparotomy for surgical reduction or resection. Mortality rate ranges from $10 \%$ to $50 \%$ (4).

\section{Case Report}

A 60 yrs old male presented with weight loss, pain abdomen and anorexia of 2 months duration. On examination, he was pale, thin and emaciated. Abdominal examination was normal. Endoscopic examination revealed an ulceroproliferative growth in the fundus of the stomach extending towards the lesser curvature. Endoscopic biopsy from the lesion was suggestive of adenocarcinoma. Computerised tomography scan of the abdomen showed presence of a growth in the fundus of stomach, extending towards the lesser curve (Figure 1). Patient underwent total gastrectomy with D2 nodal dissection and Roux-en-Y oesophagojejunostomy. Post-operative recovery was uneventful and was discharged on $12^{\text {th }}$ postoperative day. Patient got readmitted with pain abdomen, vomiting and melaena for 7 days in the $4^{\text {th }}$ week after surgery. He had a temperature of $99.0^{\circ} \mathrm{F}$, pulse rate of 98 beats $/ \mathrm{min}$, blood pressure of $110 / 78 \mathrm{~mm} \mathrm{Hg}$, respiration rate of 18 breaths/min, and oxygen saturation of $96 \%$ on room air. His cardiac and pulmonary functions were normal. His abdomen was soft, minimal abdominal distension was present with normal bowel sounds. There was mild tenderness on palpation in the periumbilical area with no obvious palpable lump. Laboratory tests showed anemia (hemoglobin of 9.8 gm $\%$ ) and an elevated white blood cell count of 14,500 per microliter (normal range:4,500-11,000 per microliter). Renal functions, electrolytes, liver function tests, and pancreatic enzymes were all within normal limits.

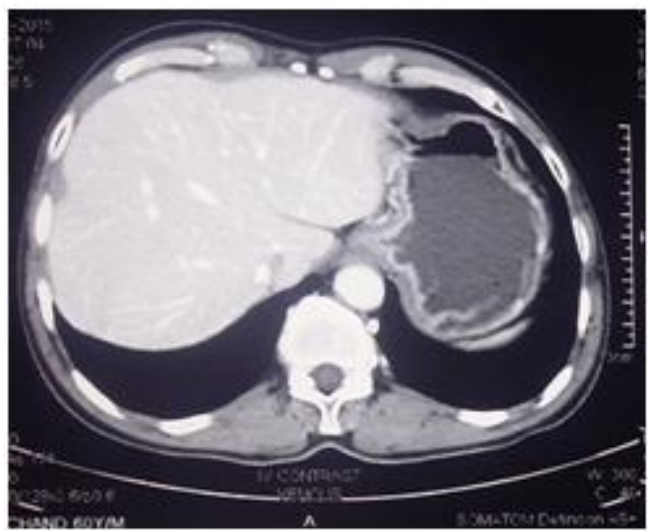

Figure 1

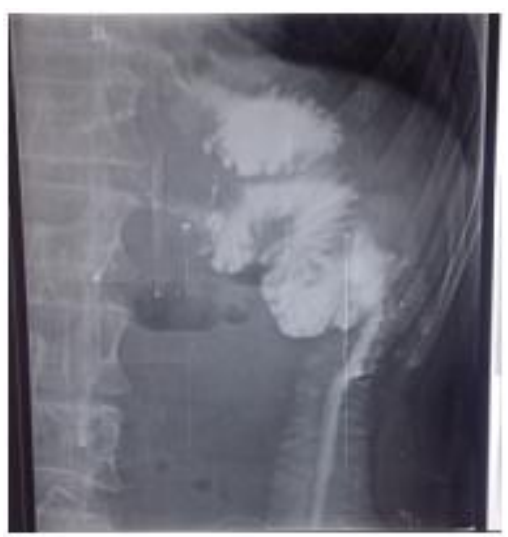

Figure 2 


\section{International Journal of Science and Research (IJSR) \\ ISSN (Online): 2319-7064}

Index Copernicus Value (2015): 78.96 | Impact Factor (2015): 6.391

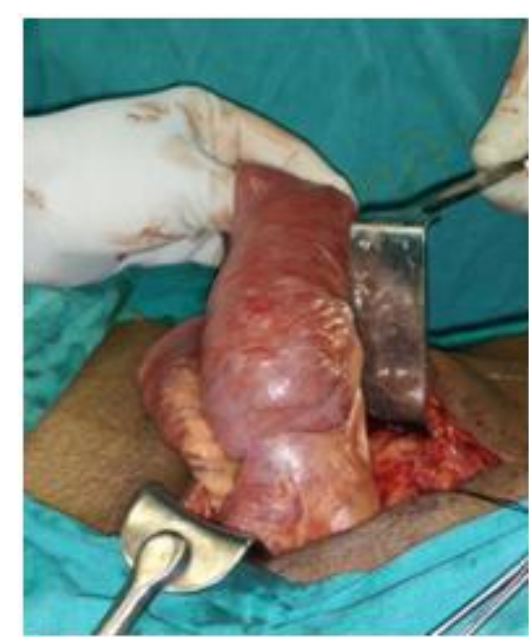

Figure 3

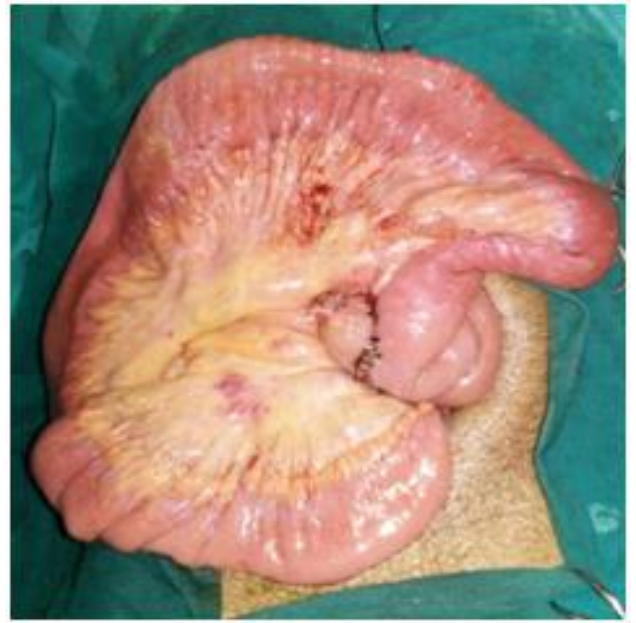

Figure 4

He was treated conservatively for 48 hours with proton pump inhibitors, antiemetics, antibiotics and antispasmodics. But he did not show good response to medical management. Barium follow through was suggestive of intestinal intussusception (Figure 2). He was taken up for emergency laparotomy which revealed jejuno-jejunal intussusception with minimal congestion in the intussusceptum in the common jejunal limb beyond jejunojejunostomy (Figure 3 and 4). Retrograde reduction of the intussusception followed by peritoneal toileting done and abdomen was closed in a single layer. Patient improved and recovered well.

\section{Discussion}

Adult intussusception occurs most often in the small bowel and is classified on the basis of its location. It can be categorized as enteroenteric (small bowel only), colocolic (large bowel only), ileocolic (terminal ileum prolapses within the ascending colon), or ileocecal (ileocecal valve is the lead point) (5). Brayton et al., in their study of 745 surgically diagnosed adult intussusceptions, $52 \%$ were found in the small intestine (39\% enteroenteric, 13\% ileocolic) and $38 \%$ in the large intestine (17\% ileocecal, $17 \%$ colocolic, $4 \%$ appendiceal) (5). This study speaks about the incidence of intussusception in normal adults with underlying malignancy, unlike our case where the patient presented with intussusception after total gastrectomy (postoperatively). Kshirsagar AY et al., had earlier reported retrograde jejuno-gastric intussusception after Billroth gastrectomy (3). In post-operative intussusception, the duration between the previous surgery and presenting symptoms can vary from 5 days to 35 years (6). Clinical presentations can be: A. Acute: present as an acute intestinal obstruction; B. Chronic recurrent: present as recurrent episodes of abdominal pain, relieved by intermittent vomiting. C. Acute postoperative: start by the fourth or fifth postoperative day and it has a tendency to reduce spontaneously (7). Various factors have been incriminated in post-operative intussusception, such as hyperacidity, adhesions around the suture lines, shortening of jejunal mesentery, long afferent loop, jejunal spasm with abnormal motility, increased intra-abdominal pressure, and retrograde peristalsis, etc. (8).

Diagnosis of intussusception in adults, even with history of the previous surgery, is difficult. The classical symptoms are absent in adults. High index of suspicion is required for early diagnosis as mortality increases from $10 \%$ to $50 \%$ with a delay of 48 hours of surgery in acute intussusceptions (3). Plain $\mathrm{X}$ - ray of abdomen may show dilated stomach or jejunum with a homogenous mass. A water-soluble upper gastrointestinal contrast study reveals a "coiled-spring" appearance of the intussusception. Ultrasonography shows a tubular mass with peristalsis and computed tomography (CT) scan will show intussusception (9). Upper gastrointestinal endoscopy helps in the diagnosis and can be therapeutic in reducing the retrograde jejuno-gastric intussusception (9). In our case, diagnosis was confirmed on barium contrast study and Computerised tomography abdomen. Immediate surgical intervention is required to avoid development of gangrene and to prevent morbidity and mortality. Reduction by gentle traction should always be attempted. If there is gangrenous bowel, then resection is mandatory. Additional procedures to prevent recurrence of intussusception, such as fixing the jejunal loops together or to the colon or mesocolon with or without enterostomy, are not warranted because of low chance of recurrence. The high mortality associated with this condition is usually due to delay in diagnosis, leading to gangrene of jejunal loops and septicemic shock (10).

\section{Conclusion}

Post-operative jejuno-jejunal intussusception is a rare but known complication after gastrectomy in adults. High index of suspicion is necessary for diagnosis and early surgical intervention is required to prevent morbidity and mortality.

\section{References}

[1] Marinis A, Yiallourou A, Samanides L, et al. Intussusception of the bowel in adults: a review. World J Gastroenterol 2009; 15(4):407-11.

[2] Marx WJ Reduction of jejunogastric intussusceptions during upper gastrointestinal examination. Am J Roentgenol. 1978; 131:334-35.

[3] Kshirsagar AY, Shinde SL, Langade YB, Pujari SG, Jeur AS. Retrograde jejunogastric intussusception with strangulation after posterior gastrojejunostomy. MJAFI 2008; 64:282-83 


\section{International Journal of Science and Research (IJSR) \\ ISSN (Online): 2319-7064}

Index Copernicus Value (2015): 78.96 | Impact Factor (2015): 6.391

[4] Teng Lu, MD; Yi-mei Chng, MD. Adult Intussusception. Perm J Winter 2015; 19(1):79-81

[5] Brayton D, Norris WJ. Intussusception in adults. Am J Surg 1954; 88(1):32-43.

[6] Hammond N, Miller FH, Dynes M Intussusception into the enteroanastomosis after billroth II gastrectomy and Roux-en-y jejunostomy: sonographic and CT findings. AJR 2001; 177:624-26.

[7] Zenooz NA, Holz SP, Robbin MR Jejunogastric intussusception: a case report with the review of literature. Emerg Radiol 2007; 13:265-67.

[8] Salem MH, Coffman SE, Postlethwait RW. Retrograde intussusception at the gastrojejunal stoma. Ann Surg 1959; 150:864-87.

[9] Loi CM, Huang SY, Chen YD, Chen SD, Wu JM, Chen KH. Retrograde jejunogastric intussusception: A case report and review of the literature. Asian J Surg 2014 2014 Jun 14. pii: S1015-9584(14)00050-5. doi: 10.1016/j.asjsur.2014.04.001. [Epub ahead of print] PubMed PMID: 24938859

[10] Sumitoj Singh, Arvinder Singh1, Suman Bhagat1, Baldev Singh. Retrograde Jejuno - gastric Intussusception, Niger J of Surg. 2016; 21(1):70-72 of Surgery Jan-Jun 2015 | Volume 21 | Issue 1

\section{Legends}

Fig. 1: CT scan showing carcinoma of stomach with wall thickness in the fundus of stomach.

Fig. 2: Barium contrast study showing the coiled spring appearance of the jejunum.

Fig. 3: Intraoperative picture of jejuno-jejunal intussusception with congestion of intussusceptum

Fig. 4: Intraoperative picture after reduction of jejunojejunal intussusception. 